\title{
Asteroseismology of the $\beta$ Cephei star $\nu$ Eridani - III. Extended frequency analysis and mode identification
}

\author{
J. De Ridder, ${ }^{1 \star}$ J. H. Telting, ${ }^{2}$ L. A. Balona,${ }^{3}$ G. Handler, ${ }^{4}$ M. Briquet, ${ }^{1}$ \\ J. Daszyńska-Daszkiewicz, ${ }^{1,5}$ K. Lefever, ${ }^{1}$ A. J. Korn, ${ }^{6}$ U. Heiter ${ }^{6}$ and C. Aerts ${ }^{1}$ \\ ${ }^{1}$ Instituut voor Sterrenkunde, K. U. Leuven, Celestijnenlaan 200B, B-3001 Leuven, Belgium \\ ${ }^{2}$ Nordic Optical Telescope, Apartado 474, 38700 Santa Cruz de La Palma, Spain \\ ${ }^{3}$ South African Astronomical Observatory, PO Box 9, Observatory 7935, Cape Town, South Africa \\ ${ }^{4}$ Institut für Astronomie der Universität Wien, Türkenschanzstr. 17, 1180 Wien, Austria \\ ${ }^{5}$ Astronomical Institute of Wroctaw University, ul. Kopernika 11, 51-622 Wroctaw, Poland \\ ${ }^{6}$ Department of Astronomy and Space Physics, Uppsala Astronomical Observatory, Box 515, SE-75120 Uppsala, Sweden
}

Accepted 2004 March 2. Received 2004 March 1; in original form 2004 February 6

\begin{abstract}
Using the large photometric and spectroscopic data sets of the $v$ Eridani multisite campaign given in our two recent papers (Aerts et al. and Handler et al.), we present an extended frequency analysis and a photometric mode identification. For the extended frequency analysis, we used an improved radial velocity time series, the second-moment time series and the line profiles themselves. In the radial velocity time series, we can now detect an additional pulsation frequency that was previously only found in photometric time series. We also report several new candidate pulsation frequencies. For seven frequencies, the photometric mode identification indicates that they belong to a radial mode and six dipole modes, and for three frequencies the degree $\ell$ could not be unambiguously determined. We also placed $v$ Eri in the HertzsprungRussell diagram by determining $T_{\text {eff }}$ using Geneva plus Strömgren photometric calibrations, spectral energy distribution fitting, by non-local thermodynamic equilibrium hydrogen, helium and silicon line profile fitting, and by determining $\log \left(L / \mathrm{L}_{\odot}\right)$ using the Hipparcos parallax and an $\mathrm{H} \beta$ calibration.
\end{abstract}

Key words: techniques: photometric - techniques: spectroscopic - stars: early-type - stars: individual: $v$ Eridani - stars: oscillations - stars: variables: other.

\section{INTRODUCTION}

The massive B-type $\beta$ Cephei stars are of particular asteroseismic interest. After all, they will be the next-generation supernovae, thereby chemically enriching our environment. Modelling their evolution, however, requires a thorough understanding of their convective core and of internal rotational mixing, two topics about which there is currently very limited knowledge. Using low-degree pulsation modes, which have very deep turning points, to probe this convective core currently seems the best way to resolve this problem.

The pulsation periods of $\beta$ Cephei stars (3-8 h) are, however, significantly longer than those of $\delta$ Scuti stars or white dwarfs, making them more challenging targets to detect pulsational frequencies. This is why, from 2002 October until 2003 January, a large multisite and multitechnique campaign was set up for a $\beta$ Cephei star. More than 2000 high-resolution spectra and 3000 photometric measurements in three passbands were obtained for $v$ Eridani (HD 29248, B2III). The data and a frequency analysis are presented in Handler

${ }^{\star}$ E-mail: joris.deridder@ster.kuleuven.ac.be et al. (2004, hereafter Paper Ia) and Aerts et al. (2004, hereafter Paper Ib). The present paper exploits these data sets further.

Successful seismic modelling involves not only detecting pulsation frequencies, but also mode identifications. Knowing the degree $\ell$ significantly reduces the number of possible mode matches, and can therefore help to narrow down the set of candidate models. In addition, it is essential to know whether we are dealing with an $m=0$ mode, as the zonal modes are not affected by rotation in the first-order approximation.

In this paper we present the final frequency analysis results (Section 2) of both the spectroscopic and the photometric time series, as well as mode identification results (Section 4). In addition we determined the basic stellar parameters to put $v$ Eridani in the Hertzsprung-Russell (HR) diagram (see Section 3). These results serve as the starting point for stellar modelling papers (Pamyatnykh, Handler \& Dziembowski 2004; Ausseloos et al., in preparation).

\section{FREQUENCY ANALYSIS}

The time series of $v$ Eri presented in Papers Ia and $\mathrm{Ib}$ are the largest ever collected for a $\beta$ Cephei star, with the sole purpose of detecting 
as many pulsational frequencies as possible. In what follows we extend the frequency analysis done in the previous papers, by a reanalysis of the radial velocity time series, and for the first time a frequency analysis of the line profile time series.

\subsection{The radial velocity}

We carefully redetermined the moments (see Aerts, de Pauw \& Waelkens 1992, for a definition) of each of the three Si lines of the Si III triplet around $456 \mathrm{~nm}$. In the intervals [455.0, 455.6] nm, [456.4, 457.2] $\mathrm{nm}$ and [457.2, 457.75] $\mathrm{nm}$ around respectively the Si III $455.3 \mathrm{~nm}$, the Si III $456.8 \mathrm{~nm}$ and the Si III $457.5 \mathrm{~nm}$ lines, we first locally renormalized the spectrum and then selected the part that should not be regarded as continuum. This part contains the spectral line as well as a bit of the continuum that is difficult to distinguish from the far wings of the line. The remaining part of the interval was used to compute the standard deviation $\sigma$ of the normalized flux noise. The region of the spectrum with points at least $2 \sigma$ in flux away from the continuum was then extracted as the line profile, of which the normalized moments were consequently computed. The integration boundaries of the moments are thus dynamically determined depending on the signal-to-noise $(\mathrm{S} / \mathrm{N})$ ratio $\left(\equiv \sigma^{-1}\right)$ of the spectrum. The corresponding integration interval is much smaller than the wavelength intervals stated above. The advantage is a considerable noise reduction in the moments time series. As the main mode of $v$ Eri has a large amplitude $\left(\sim 22 \mathrm{~km} \mathrm{~s}^{-1}\right)$, the spectral lines show a rather large periodic Doppler shift. A fixed wavelength integration range would therefore have to be taken quite large to ensure that the entire line profile is always included. This would also mean the inclusion of noisy continuum on the red side when the line shows a blueshift and vice versa, which degrades the accuracy of the moments.

Note that, as in Paper Ib, we compute the radial velocity with the first moment of the line profile, and not by the central wavelength of a fitted Gaussian, as the Si line profiles are sometimes rather skewed. We also did not use the wavelength of the minimum flux point as this could lead to frequency harmonics in the $v_{\text {rad }}$ data regardless of whether the star pulsates linearly or not.

After removing noisy data points $(\mathrm{S} / \mathrm{N}<150)$ and outliers, we retained 1740,1874 and 1827 data points for respectively the Si III $455.3,456.8$ and $457.5 \mathrm{~nm}$ lines. We did a careful frequency analysis of these $v_{\text {rad }}$ data and we tabulate the results later in this section. We find all the 'independent' frequencies mentioned in Paper Ib, and in addition we now find three (instead of only two) frequency peaks of the multiplet around $6.24 \mathrm{~d}^{-1}$. We also find most of the "combination' frequencies stated in Paper Ib, plus some additional ones. Just as in Papers Ia and Ib, we identify a combination frequency as a frequency outside the usual $\beta$ Cephei frequency interval of $[3,8] \mathrm{d}^{-1}$, and which can be written as a linear combination of two or more independent frequencies within the estimated observational errors. During multifrequency solution determinations, these combination frequencies are fixed to their exact linear combination. We realize, however, that this approach is not without risk, as it might not be impossible that a linearly stable high-order eigenfrequency close to but not exactly equal to a linear combination frequency might be excited due to non-linear effects. In such a case, fixing the combination frequencies would lead to a biased estimate of the independent frequencies. We nevertheless choose to fix the combination frequencies as it seems unlikely that all or even most of them would be real eigenfrequencies. The alternative, i.e. introducing extra free parameters, would then lead to overfitting and thus to an excessive variance of the estimated independent frequencies.
We made an effort to determine the optimal multifrequency solution in an $N$-dimensional box with edges $2 \Delta v$ around the initial estimate. One way of doing this is to make an $N$-dimensional grid in the box, with mesh size $\delta v$, and verify the performance of the frequency solution in each of the $(2 \Delta v / \delta v+1)^{N}$ grid points. This number of grid points is, however, computationally unfeasible for $v$ Eri, for which we have $N=8$ and $\Delta v=10^{-2} \mathrm{~d}^{-1}$ and for which we would like to have $\delta v=10^{-4} \mathrm{~d}^{-1}$. We therefore resorted to $\mathrm{N}$-dimensional conjugate directions minimization (see e.g. Press et al. 1992), which we choose because there is no need for derivatives. The function to minimize was the $\chi^{2}$ function with eight dependent and 12 combination frequencies in the case of a spectroscopic data set, and nine dependent frequencies and 14 combination frequencies in the case of a photometric data set. This method seemed to perform quite well in a reasonable amount of time. Unfortunately, the window function of the spectroscopic time series has local maxima at $v=0.0023$ and $0.0046 \mathrm{~d}^{-1}$, which may have led the algorithm to an alias frequency. We therefore also systematically checked whether the frequency combination

$\left\{v_{1}+n_{1} \times 0.0023, v_{2}+n_{2} \times 0.0023, \ldots, v_{8}+n_{8} \times 0.0023\right\}$,

where $\left\{v_{i}\right\}$ is the solution after minimization and $n_{i} \in\{0, \pm 1, \pm$ $2\}$, has a better $\chi^{2}$ value. The results of this optimization approach are given in Table 1, where we also give for each frequency the amplitude and phase together with their estimated uncertainties.

In fact, one reason for giving Table 1 in addition to table 2 of both Papers Ia and $\mathrm{Ib}$ is to provide a better feeling for the uncertainties involved. We underline that the error estimates on the frequencies given in Papers Ia and Ib are formal estimates (as clearly stated), derived under the assumption of an equidistant time series of a monoperiodic signal with uncorrelated noise. The different values of the derived frequencies for the different Si lines and for the different Strömgren passbands in Table 1 provides another way to assess the uncertainties on the frequencies. The uncertainties on the amplitudes and the phases were computed, as usual, with the local curvature of the $\chi^{2}$ function where we assumed the frequencies to be known. The derived values of the amplitudes and phases (the latter are not given in Papers Ia and Ib) for the different Si lines provide another view on the uncertainties involved. Especially the phases seem to be rather inaccurately determined. We note that both the phase and its uncertainty depend on $t_{0}$ as well as on the exact value of the frequency.

Concerning the close frequencies around $6.24 \mathrm{~d}^{-1}$, we easily find the frequencies $v_{6}$ and $v_{8}$ in the new radial velocity time series; these were also detected in the photometric time series. The frequency $v_{8}$ is new for the spectroscopic data, as it was not found in the $v_{\text {rad }}$ data of Paper Ib. A peak at the position of $v_{7}$ (a frequency that was clearly detected in Paper Ib, and mentioned as a possible photometric signal in Paper Ia) is seen in the radial velocity time series of all three $\mathrm{Si}$ lines, but shows much less power than mentioned in Paper Ib, as can be read from Table 1. Much of the evidence for $v_{7}$ therefore comes from both Papers Ia and Ib instead of from our new radial velocity time series.

At this point we would like to mention that we did a frequency analysis not only of the first moment, but also of the second moment $\left\langle v^{2}\right\rangle$. The motivation to do so is the $\beta$ Cephei star $\beta$ Crucis, for which some pulsation frequencies were found in the higher moments but not in $\langle v\rangle$ (Aerts et al. 1998), and which were later confirmed by satellite (WIRE) data (Cuypers et al. 2002). However, a frequency analysis of the second moment is notoriously difficult because of the many possible cross-term frequencies. If there are $N$ frequencies $v_{i}$ detected in the first moment, there can be $N(N$ 
Table 1. Estimates of the frequencies, amplitudes and phases of the modes found in the radial velocity and photometric time series. Each frequency was assigned a unique identification. The signal is always written as $C+\sum_{i=1}^{N} A_{i} \sin \left[2 \pi v_{i}\left(t-t_{0}\right)+\varphi_{i}\right]$, where $t_{0}=$ HJD 2452000 . Eight independent and 12 combination frequencies were found in the $v_{\text {rad }}$ data, and nine independent and 14 combination frequencies were found in the photometric data. The combination frequencies were always fixed to their exact linear combination of independent frequencies. The uncertainties on the last given digit of the amplitudes and phases are given in parentheses.

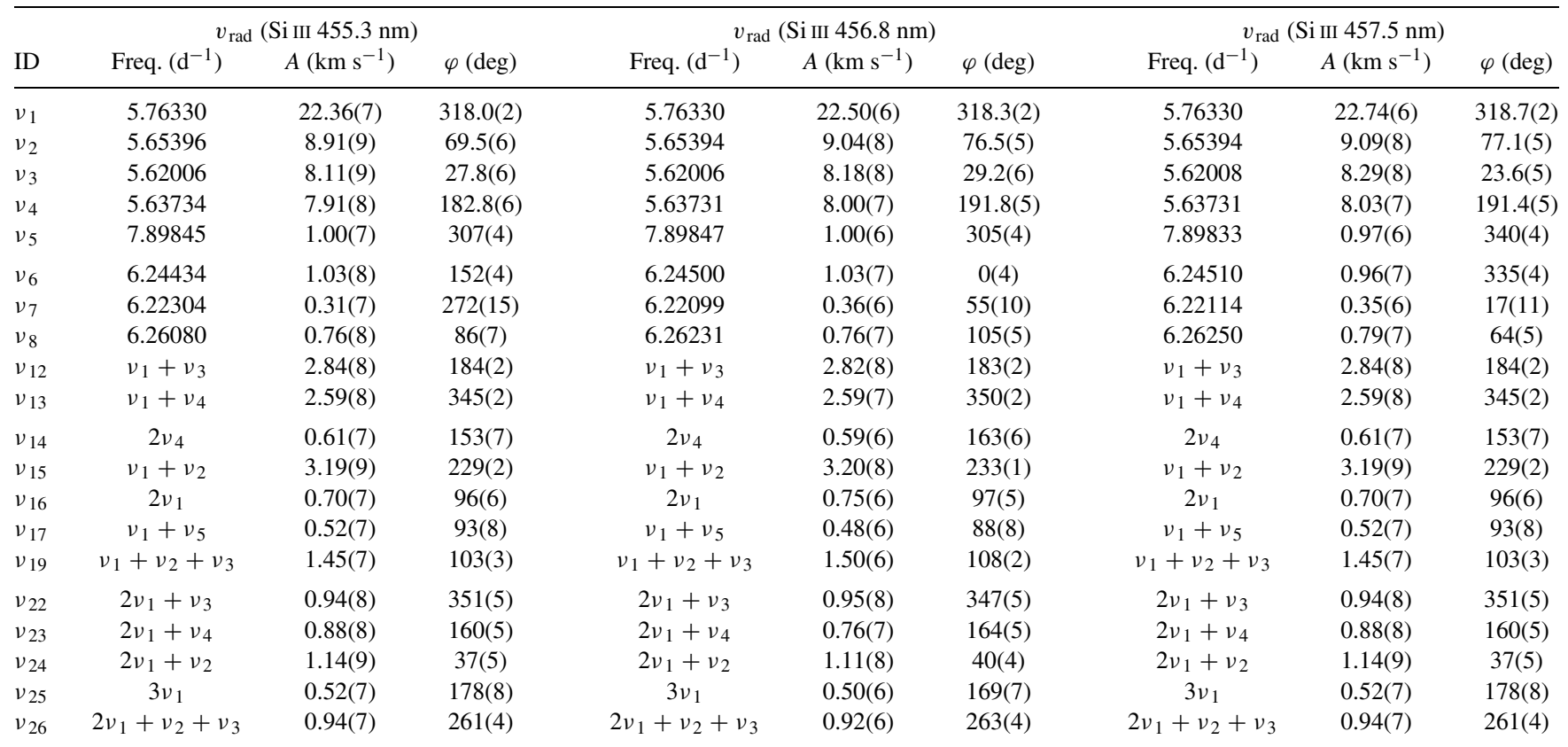

\begin{tabular}{|c|c|c|c|c|c|c|c|c|c|}
\hline \multirow[b]{2}{*}{ ID } & \multicolumn{3}{|c|}{ Strömgren $u$} & \multicolumn{3}{|c|}{ Strömgren $v$} & \multicolumn{3}{|c|}{ Strömgren $y$} \\
\hline & Freq. $\left(d^{-1}\right)$ & $A$ (mmag) & $\varphi(\operatorname{deg})$ & Freq. $\left(d^{-1}\right)$ & $A$ (mmag) & $\varphi(\mathrm{deg})$ & Freq. $\left(d^{-1}\right)$ & $A$ (mmag) & $\varphi(\operatorname{deg})$ \\
\hline$v_{2}$ & 5.65391 & $37.9(2)$ & 172.7(3) & 5.65396 & $26.5(1)$ & $162.0(3)$ & 5.65393 & 25.1(1) & $168.1(3)$ \\
\hline$v_{4}$ & 5.63715 & $32.2(2)$ & $319.8(4)$ & 5.63720 & $22.4(2)$ & $307.8(4)$ & 5.63718 & 21.1(1) & $313.6(4)$ \\
\hline$v_{5}$ & 7.89757 & $4.3(2)$ & $246(3)$ & 7.89769 & $3.1(1)$ & $219(3)$ & 7.89779 & $2.9(1)$ & $195(2)$ \\
\hline$v_{6}$ & 6.24352 & $3.9(2)$ & $61(3)$ & 6.24406 & $2.6(1)$ & $307(3)$ & 6.24326 & $2.6(1)$ & $114(3)$ \\
\hline$v_{10}$ & 0.43235 & $5.5(2)$ & $187(2)$ & 0.43190 & $3.2(1)$ & $289(2)$ & 0.43232 & $3.2(1)$ & $192(2)$ \\
\hline$v_{11}$ & $v_{2}+v_{3}$ & $2.8(2)$ & $11(4)$ & $v_{2}+v_{3}$ & $1.7(1)$ & $2(5)$ & $v_{2}+v_{3}$ & $1.4(1)$ & $2(5)$ \\
\hline$v_{12}$ & $v_{1}+v_{3}$ & 11.1(2) & 297(1) & $v_{1}+v_{3}$ & $7.9(1)$ & $303(1)$ & $v_{1}+v_{3}$ & $7.5(1)$ & $300(1)$ \\
\hline$v_{13}$ & $v_{1}+v_{4}$ & $10.9(2)$ & $150(1)$ & $v_{1}+v_{4}$ & $7.7(2)$ & $139(1)$ & $v_{1}+v_{4}$ & 7.1(1) & $143(1)$ \\
\hline$v_{15}$ & $v_{1}+v_{2}$ & $12.6(2)$ & $5.6(9)$ & $v_{1}+v_{2}$ & $9.0(1)$ & $354.9(9)$ & $v_{1}+v_{2}$ & $8.4(1)$ & $358.8(9)$ \\
\hline$v_{16}$ & $2 v_{1}$ & $4.5(2)$ & $183(3)$ & $2 v_{1}$ & $3.1(1)$ & $176(3)$ & $2 v_{1}$ & $2.9(1)$ & $175(3)$ \\
\hline$v_{20}$ & $v_{1}+v_{2}+v_{4}$ & $1.0(2)$ & $53(13)$ & $v_{1}+v_{2}+v_{4}$ & $0.9(1)$ & $30(10)$ & $v_{1}+v_{2}+v_{4}$ & $0.9(1)$ & $50(9)$ \\
\hline$v_{21}$ & $v_{1}+2 v_{2}$ & $0.9(2)$ & $257(14)$ & $v_{1}+2 v_{2}$ & $0.8(1)$ & $236(11)$ & $v_{1}+2 v_{2}$ & $0.6(1)$ & $253(15)$ \\
\hline$v_{22}$ & $2 v_{1}+v_{3}$ & $1.8(2)$ & $113(7)$ & $2 v_{1}+v_{3}$ & $1.4(1)$ & 106(6) & $2 v_{1}+v_{3}$ & $1.2(1)$ & $106(6)$ \\
\hline$v_{23}$ & $2 v_{1}+v_{4}$ & $1.8(2)$ & $335(7)$ & $2 v_{1}+v_{4}$ & $1.2(1)$ & $314(7)$ & $2 v_{1}+v_{4}$ & $1.1(1)$ & $313(8)$ \\
\hline$v_{24}$ & $2 v_{1}+v_{2}$ & $2.0(2)$ & $181(6)$ & $2 v_{1}+v_{2}$ & $1.4(1)$ & $162(6)$ & $2 v_{1}+v_{2}$ & $1.3(1)$ & $166(6)$ \\
\hline$v_{26}$ & $2 v_{1}+v_{2}+v_{3}$ & $1.5(2)$ & $65(7)$ & $2 v_{1}+v_{2}+v_{3}$ & $1.0(1)$ & $67(8)$ & $2 v_{1}+v_{2}+v_{3}$ & $0.9(1)$ & $69(8)$ \\
\hline
\end{tabular}

+1 ) frequencies present in the second moment: besides $v_{i}$ also $2 v_{i}, v_{i}+v_{j}$ and $v_{i}-v_{j}$ (Aerts 1996). In the case of $v$ Eri this would imply that we can expect 420 frequencies, although not all of them need to have an amplitude above the noise level. The power spectrum of the second-moment time series of the Si III $(456.8 \mathrm{~nm})$ line indeed shows many peaks, and we do not list them here for the sake of brevity. The bottom line of our analysis is that, besides the many cross-term frequencies, we did not find any convincing new frequencies.

\subsection{The line profiles}

We continued our quest for frequencies by doing a frequency analysis on the line profiles of the Si III 455.3 and $456.8 \mathrm{~nm}$ lines. Indeed, 


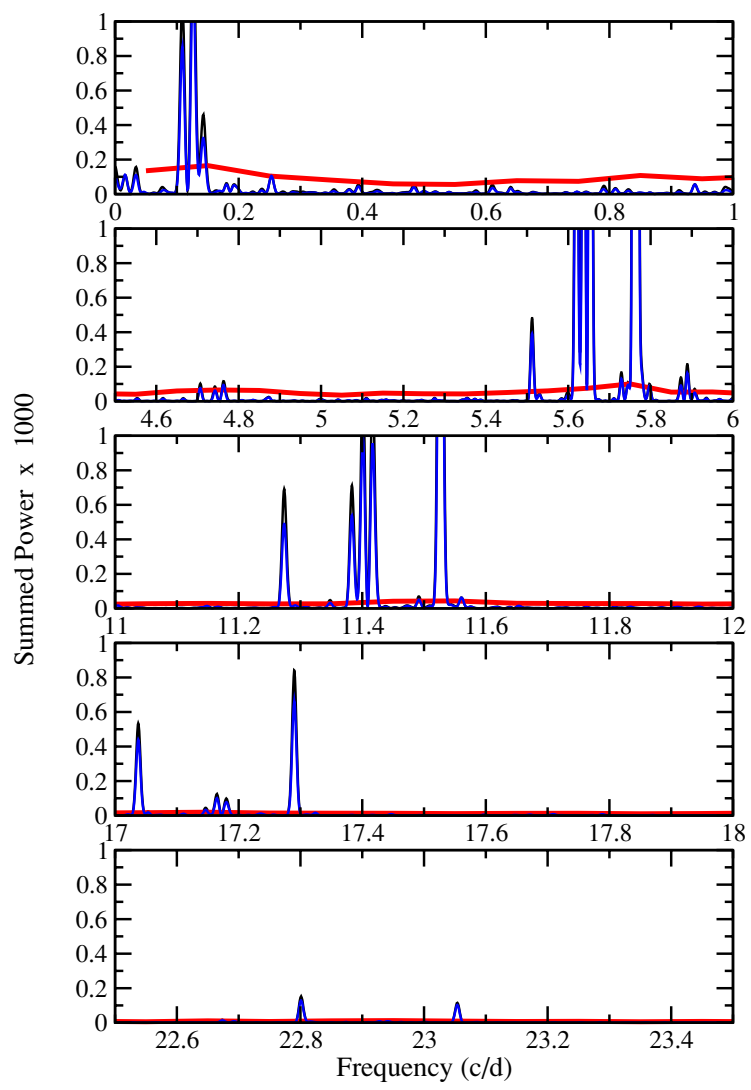

Figure 1. The power spectra of the Si III 455.3 and $456.8 \mathrm{~nm}$ line profiles, obtained by summing the power at each wavelength point in the intervals [455.16, 455.40] $\mathrm{nm}$ and $[456.678,456.922] \mathrm{nm}$, respectively. The power spectra are plotted on top of each other, and can hardly be distinguished. The CLEAN algorithm with gain parameter $g=0.2$ was used. The thick almost horizontal line denotes the $1 \sigma$ noise level of the non-CLEAN-ed Fourier power spectrum after prewhitening the time series with the 20 frequencies mentioned in Table 2, and was approximated at each frequency by the average power in a $0.1 \mathrm{~d}^{-1}$ interval around that frequency.

several examples of $\beta$ Cephei stars exist for which some pulsation frequencies are clearly detected in the line profiles but not in the radial velocity data (e.g. $\varepsilon$ Cen, Schrijvers, Telting \& Aerts 2004).

We considered normalized flux time series at each wavelength point in the intervals [455.16, 455.40] $\mathrm{nm}$ and [456.678, 456.922] nm. We used the CLEAN algorithm (Roberts, Lehar \& Dreher 1987) to search for frequencies but systematically verified with classical Fourier analysis that our results are not numerical artefacts. The frequency analysis was done in the interval $[0,30] \mathrm{d}^{-1}$ with a frequency step of $2 \times 10^{-6} \mathrm{~d}^{-1}$. Consequently, we summed the power over all the wavelength bins to obtain an overall power spectrum. The result is shown in Fig. 1. In the corresponding Table 2 we list the 20 frequencies that are clearly present in both silicon line profiles. We also list each time the suspected identification, which is always either one of the independent frequencies also found in the radial velocity, or a linear combination of these independent frequencies. Besides these frequencies we also consider the low-amplitude frequencies 4.707 and $4.742 \mathrm{~d}^{-1}$, worth comparing with theoretically predicted frequencies.

In Table 2 we list two frequency combinations as possible identifications for the frequencies 5.51 and $5.89 \mathrm{~d}^{-1}$. We consequently asked ourselves the following question: As we have nine genuine
Table 2. All frequency peaks that are clearly present in both Si III 455.3 and $456.8 \mathrm{~nm}$ line profiles. We also list the suspected identification, where we have used the same notation as in Table 1.

\begin{tabular}{lcc}
\hline $\begin{array}{l}\text { Si III } 455.3 \mathrm{~nm} \\
\text { Freq. }\left(\mathrm{d}^{-1}\right)\end{array}$ & $\begin{array}{c}\text { Si III } 456.8 \mathrm{~nm} \\
\text { Freq. }\left(\mathrm{d}^{-1}\right)\end{array}$ & $\begin{array}{c}\text { Possible } \\
\text { Identification }\end{array}$ \\
\hline 0.10880 & 0.10886 & $v_{1}-v_{2}$ \\
0.12630 & 0.12622 & $v_{1}-v_{4}$ \\
0.14270 & 0.14268 & $v_{1}-v_{3}$ \\
5.51228 & 5.51224 & $2 v_{4}-v_{1}$ \\
5.61986 & 5.61986 & $v_{3}$ \\
5.63760 & 5.63762 & $v_{4}$ \\
5.65384 & 5.65390 & $v_{2}$ \\
5.76334 & 5.76336 & $v_{1}$ \\
5.88910 & 5.88910 & $2 v_{1}-v_{4}$ \\
11.27368 & 11.27364 & $v_{11}$ \\
11.38330 & 11.38326 & $v_{12}$ \\
11.40108 & 11.40104 & $v_{13}$ \\
11.41688 & 11.41688 & $v_{15}$ \\
11.52670 & 11.52672 & $v_{16}$ \\
17.03752 & 17.03752 & $v_{19}$ \\
17.16478 & 17.16480 & $v_{23}$ \\
17.18004 & 17.18006 & $v_{24}$ \\
17.28994 & 17.28996 & $v_{25}$ \\
22.80086 & 22.80086 & $v_{26}$ \\
23.05362 & 23.05360 & $4 v_{1}$ \\
\hline
\end{tabular}

eigenfrequencies $v_{1}, \ldots, v_{9}$, should we not expect that almost every newly found frequency peak in the $\beta$ Cephei pulsational frequency range $\left[v_{\mathrm{b}}, v_{\mathrm{e}}\right]=[3,8] \mathrm{d}^{-1}$ can be written as some linear combination of these eigenfrequencies? Part of the answer is that it turns out that there are actually not many possible linear combinations that map frequencies from $\left[v_{\mathrm{b}}, v_{\mathrm{e}}\right]$ back into the same interval and that are at the same time not too far-fetched with respect to the linear combinations found in the Fourier spectrum of the radial velocity. Only the general combinations $2 v_{j}-v_{k}$ and $v_{j}+v_{k}-v_{i}$ seem to be acceptable. We now compute the probability that a randomly chosen frequency coincides with a combination frequency $v_{\mathrm{c}}$. Given $n$ independent frequencies, the number of possible combinations $2 v_{j}-v_{k}$ is simply $n(n-1)$, and the number of different combinations $v_{j}+v_{k}-v_{i}$ is $n(n-1)(n-2) / 2$. We consider a frequency to 'coincide' with a combination frequency $v_{c}$ if it is in the interval $\left[v_{\mathrm{c}}-\delta v, v_{\mathrm{c}}+\delta v\right]$ with $\delta v$ being the observational frequency precision. If $n$ and/or $\delta v$ is (are) sufficiently small (as in our case) we can expect that none of these intervals will overlap with another interval. All of these intervals together occupy a fraction

$\frac{\delta v}{v_{\mathrm{e}}-v_{\mathrm{b}}} n^{2}(n-1)$

of the total $\beta$ Cephei frequency range $\left[v_{\mathrm{b}}, v_{\mathrm{e}}\right]$, which is equal to the probability that a frequency randomly chosen in this interval falls into one of the intervals. Setting $n=9,\left[v_{\mathrm{b}}, v_{\mathrm{e}}\right]=[3,8] \mathrm{d}^{-1}$ and $\delta v=10^{-3} \mathrm{~d}^{-1}$ for the case of $v$ Eri, we can roughly estimate this probability to be 13 per cent, i.e. a non-negligible probability. We must note, however, that our assumption that the newly found frequency peak is independent of the confirmed eigenfrequencies might not be justified. If it is indeed a genuine eigenfrequency, the underlying pulsation physics may impose that it can or cannot be written as a linear combination of the other eigenfrequencies.

In fact, in Fig. 2 we find two indications that the frequencies 5.51 and $5.89 \mathrm{~d}^{-1}$ might indeed not be combination frequencies. This figure shows the morphology of the amplitude and the phase 

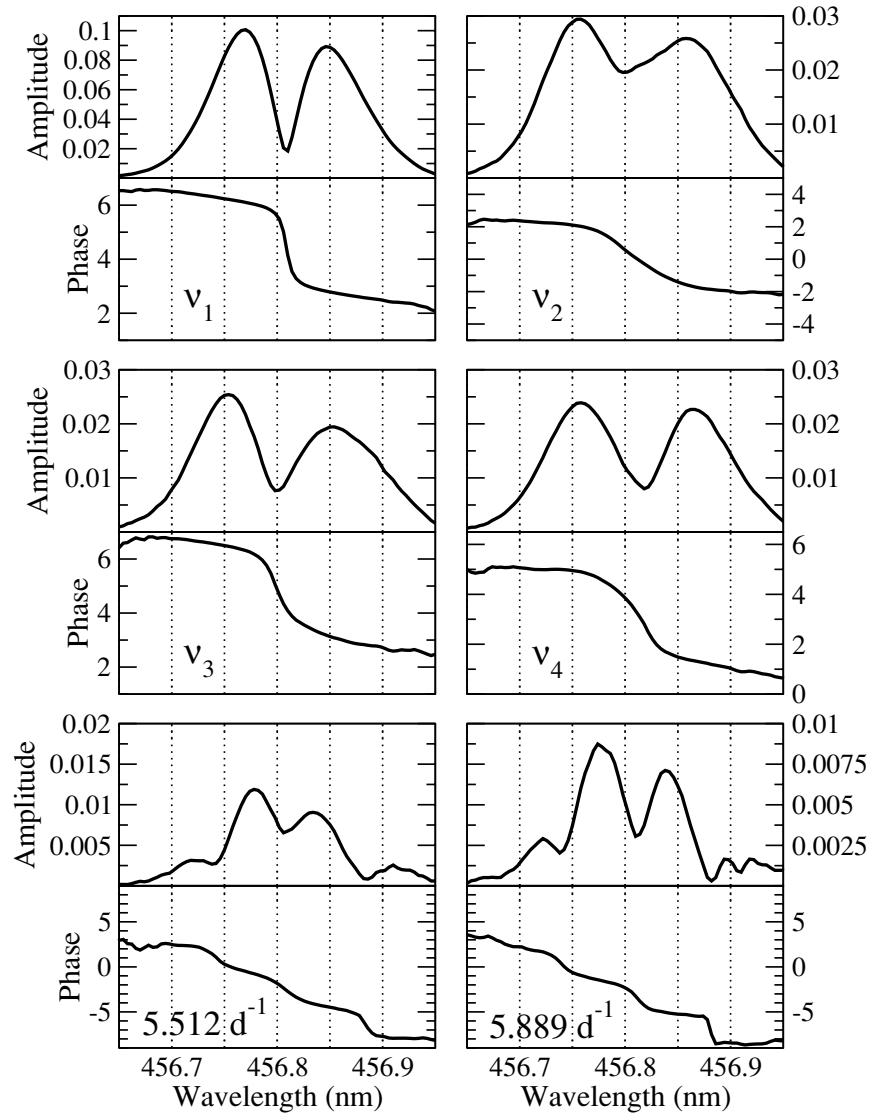
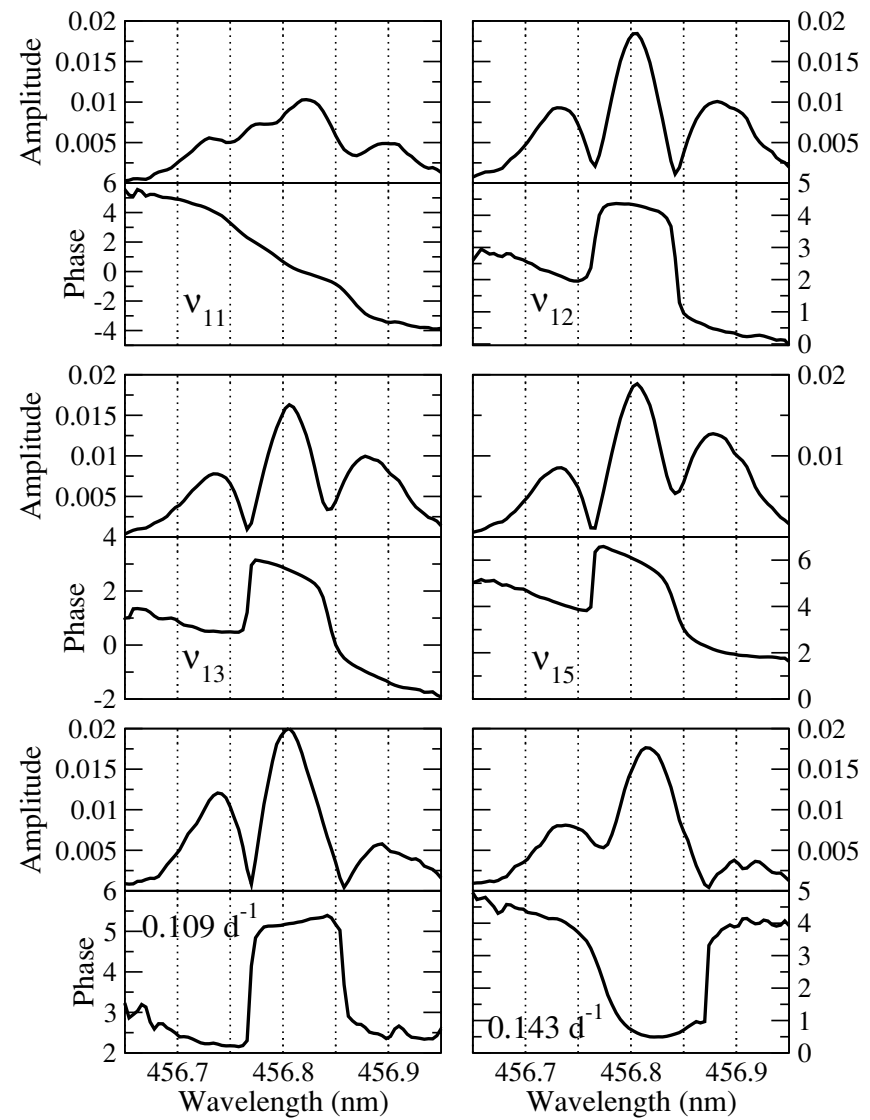

Figure 2. Amplitude and phase distributions for several frequencies, for the Si III $456.8 \mathrm{~nm}$ line. On the left-hand side the distributions of the genuine eigenfrequencies plus the frequencies 5.51 and $5.89 \mathrm{~d}^{-1}$ are shown. For comparison, on the right-hand side, we also show the distributions of some combination frequencies. The amplitudes are unitless as the line profiles are normalized. The phases are expressed in radians.

distribution of several frequencies found in the Si III $456.8 \mathrm{~nm}$ line profile time series. The amplitudes and phases were computed by fitting the flux time series at each wavelength point with a sum of 20 sines corresponding to the 20 frequencies listed in Table 2 . The amplitude and phases of these sines then make up the distributions shown in Fig. 2. We show examples of both genuine eigenfrequencies and known combination frequencies. The phase diagrams of the 5.51 and $5.89 \mathrm{~d}^{-1}$ frequencies show a monotonic phase distribution across the line profile with a large phase range. Such behaviour is typical for higher-degree modes, which would explain why we do not see the frequencies in the photometry or the radial velocity data. However, this evidence is not conclusive, as the combination frequency $v_{11}$ shows the same phase behaviour in the line profile. Another indication is that for both 5.51 and $5.89 \mathrm{~d}^{-1}$ the line profile shows more variability in the line wings than in the line core, which is also systematically true for the eigenfrequencies, while it is vice versa for the combination frequencies. Again, this evidence is not conclusive, as we do not know what the velocity distribution of a combination frequency looks like at the surface of the star, so that we also do not know what kind of line profile variations we should expect.

\section{BASIC STELLAR PARAMETERS}

For a photometric mode identification it is useful to have the position of $v$ Eri in the HR diagram. This position and its error box can also be used as the initial guess and additional constraint for further asteroseismic investigations.
Geneva photometric colours of $v$ Eri were obtained from the General Catalogue of Photometric Data (Mermilliod, Mermilliod $\&$ Hauck 1997). From these colours, the Geneva indices $X$ and $Y$ can be deduced, which are independent of interstellar extinction for hot stars like $v$ Eri. We then used the calibration of Künzli et al. (1997) to obtain $T_{\text {eff }}=23084 \pm 234 \mathrm{~K}$ and $\log g=3.88 \pm 0.19$. We emphasize that the uncertainties quoted are lower limits, as the calibration for hot stars requires the metallicity to be known a priori, but we assumed it to be solar.

Besides Geneva photometry we also used Strömgren photometry to estimate the effective temperature. We used the large literature survey already done by Hauck \& Mermilliod (1998) to obtain the following quantities for $v$ Eri: $V=3.960, b-y=-0.076, m_{1}=$ $0.068, c_{1}=0.072$ and $\beta=2.610$. With these values we can compute the following reddening-free indices (Strömgren 1966):

$\left[c_{1}\right]=c_{1}-0.2(b-y)=0.087$,

$\left[m_{1}\right]=m_{1}+0.18(b-y)=0.054$,

$[u-b]=\left[c_{1}\right]+2\left[m_{1}\right]=0.196$.

Consequently, we used the $[u-b]-T_{\text {eff }}$ calibration

$\frac{5040 \mathrm{~K}}{T_{\mathrm{eff}}}=0.1692+0.2828[u-b]-0.0195[u-b]^{2}$

of Napiwotzki, Schönberner \& Wenske (1993) to estimate $T_{\text {eff }}=$ $22500 \mathrm{~K}$.

We also estimated $T_{\text {eff }}$ by fitting the spectral energy distribution (SED) of $v$ Eri. To do so, we collected optical and infrared fluxes 

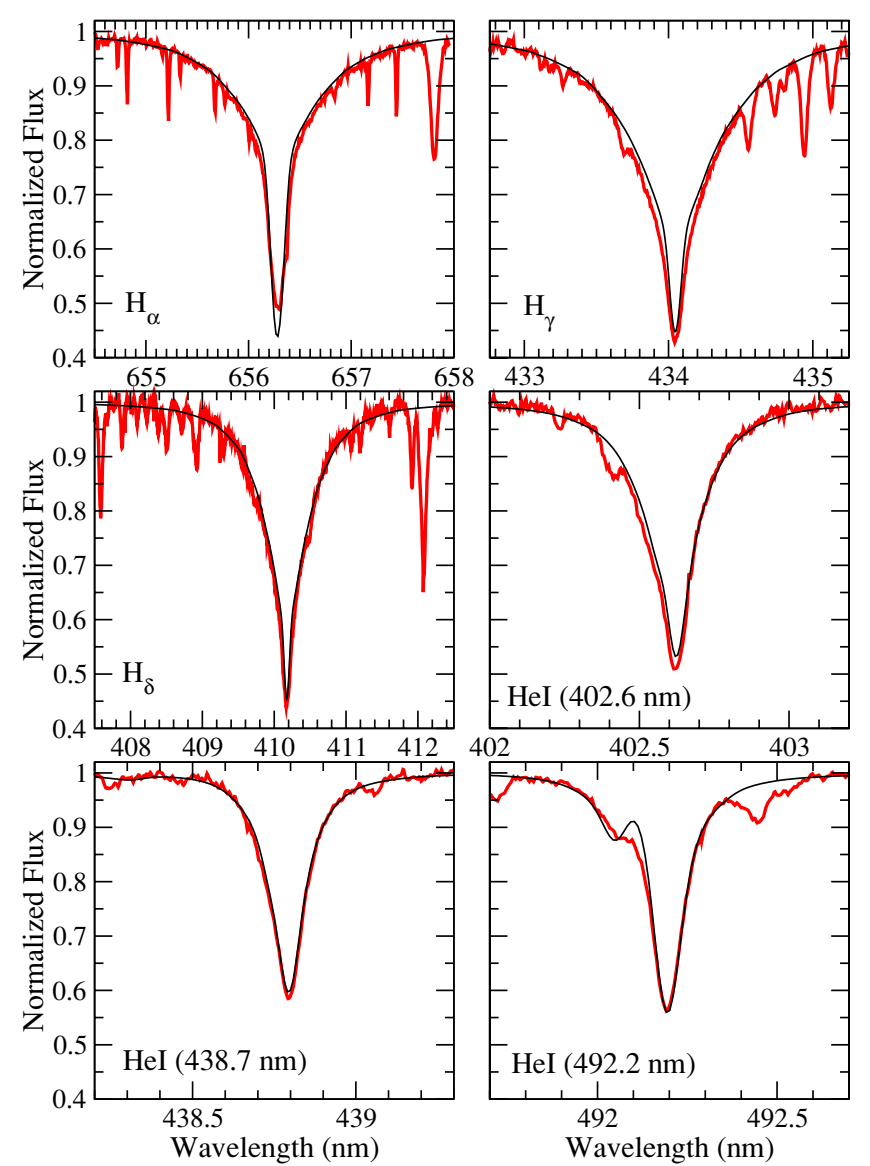
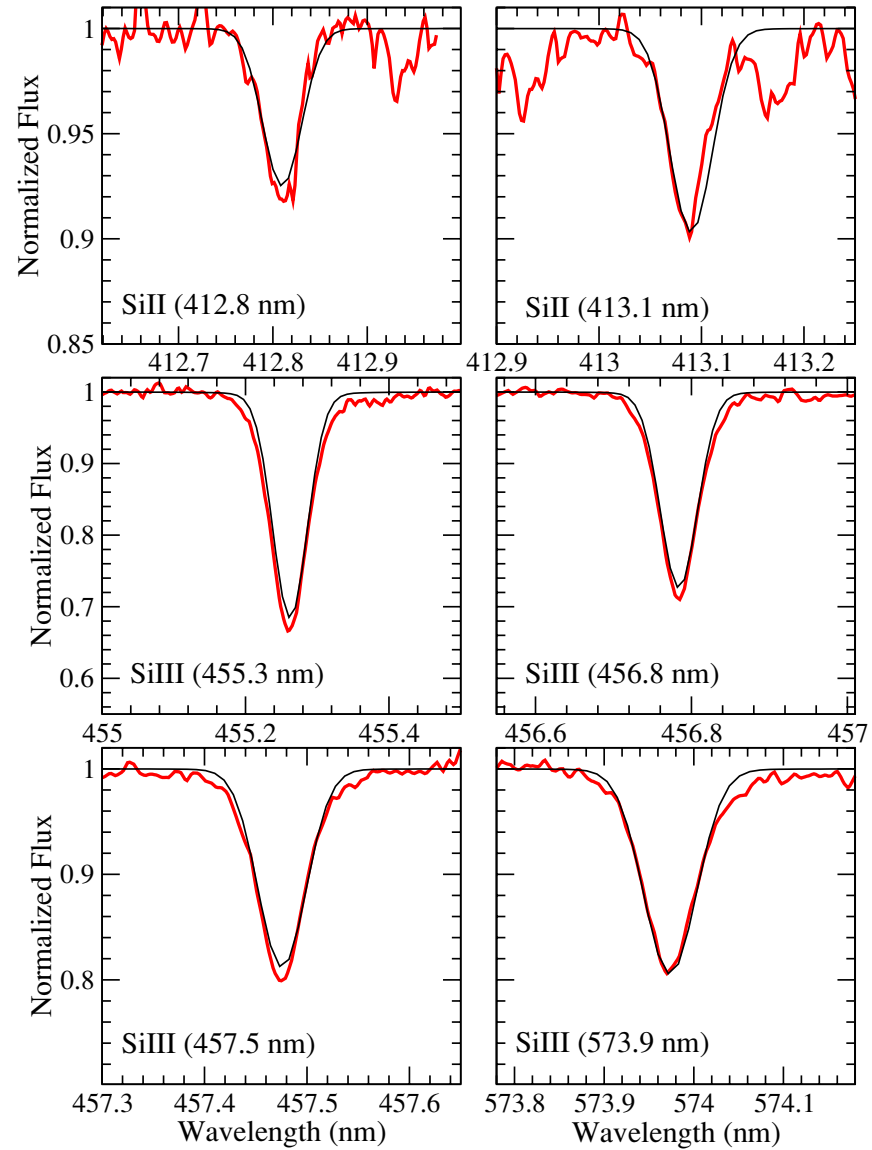

Figure 3. A selection of observational hydrogen, helium and silicon line profiles together with their best fits (smooth curves) obtained for $T_{\text {eff }}=24000 \mathrm{~K}$, $\log g=3.7$ and $n(\mathrm{He}) / n(\mathrm{H})=0.12$.

of $v$ Eri from Glushneva et al. (1992) and ultraviolet fluxes from Jamar et al. (1976). It is difficult to estimate to what extent this SED is reddened, but we tried to correct for reddening by using Fitzpatrick's (1999) mean galactic interstellar extinction law. Next, we fitted the dereddened SED with theoretical SEDs computed from local thermodynamic equilibrium (LTE) Kurucz (1994) stellar atmosphere models. The best fit was obtained for a model with $T_{\text {eff }}$ $=21900 \mathrm{~K}$. To have an idea about the uncertainty of this value, we examined the effects of small variations of the extinction or the observed ultraviolet fluxes (which are most important for our temperature determination) within their observational errors. From this, we roughly estimate the uncertainty to be about $1000 \mathrm{~K}$.

We also used the echelle spectra obtained at the Calar Alto Observatory (see Paper Ib) to make a spectroscopic estimate for $T_{\text {eff }}$. To generate a grid of theoretical line profiles we ran the spherically symmetric non-local thermodynamic equilibrium (NLTE) atmosphere code FASTWIND, first introduced by Santolaya-Rey, Puls \& Herrero (1997). The newest version of FASTWIND includes the effects of metal line blocking/blanketing (see Herrero, Puls \& Najarro 2002) as well as a consistently calculated temperature structure based on the thermal balance of electrons, except for the innermost part of the atmosphere, where a flux-correction method is applied (see Kubat, Puls \& Pauldrach 1999). We used five hydrogen lines (H $\alpha$ up to $\mathrm{H} \epsilon$ ), six He I lines (402.6, 438.7, 447.1, 471.3, 492.2 and $667.8 \mathrm{~nm})$, two Si II doublets (412.8-413.1 and 504.1-505.6 $\mathrm{nm})$, two Si III triplets (455.3-456.8-457.5 and 481.3-481.9-482.9 $\mathrm{nm})$ and two additional Si III lines (471.6 and $574.0 \mathrm{~nm}$ ). These theoretical line profiles were then compared with the least pulsationally broadened observational line profiles to search for the $T_{\text {eff }}$, $\log g$ and particle number ratio $n(\mathrm{He}) / n(\mathrm{H})$ for which the match is best. Especially the wings of the hydrogen lines and the ionization balance between Si II and Si III are useful to determine respectively the gravity and the temperature. Our best match turns out to be for the parameters $T_{\text {eff }}=24000 \mathrm{~K}, \log g=3.7$ and $n(\mathrm{He}) / n(\mathrm{H})=$ 0.12 . We estimate the uncertainties on these parameter values to be $1000 \mathrm{~K}, 0.1$ dex and 0.02, respectively. In Fig. 3 we show fits of 12 of the 23 selected spectral lines as examples. The fact that we seem to find a slight He overabundance is not too surprising as the chemical analysis of Kilian (1992) shows that half of her sample of unevolved B stars are actually He overabundant. The very slow rotation of $v$ Eri (see Paper Ib) is in fact consistent with our findings in the sense that Zboril \& North (1999) show that He-enhanced B stars are significantly slower rotators than normal B-type stars. We also refer to a recent abundance analysis of $v$ Eri of Korn \& Heiter (in preparation), who, besides $\mathrm{H}, \mathrm{He}$ and $\mathrm{Si}$ lines, also used oxygen lines to determine abundances. They find a slight He enhancement as well, although, as in our case, the uncertainties on the observed abundances cannot exclude a solar abundance. Also the derived abundances of $\mathrm{Si}$ and $\mathrm{O}$ are solar within the error bars.

Following the discussion above, we adopt conservatively $T_{\text {eff }}=$ $22900 \pm 1100 \mathrm{~K}$, in the hope that the true value is indeed contained in this rather large interval.

In order to compute the luminosity from the visual magnitude $V$, we first need to compute the interstellar extinction $A_{V}$. Although 


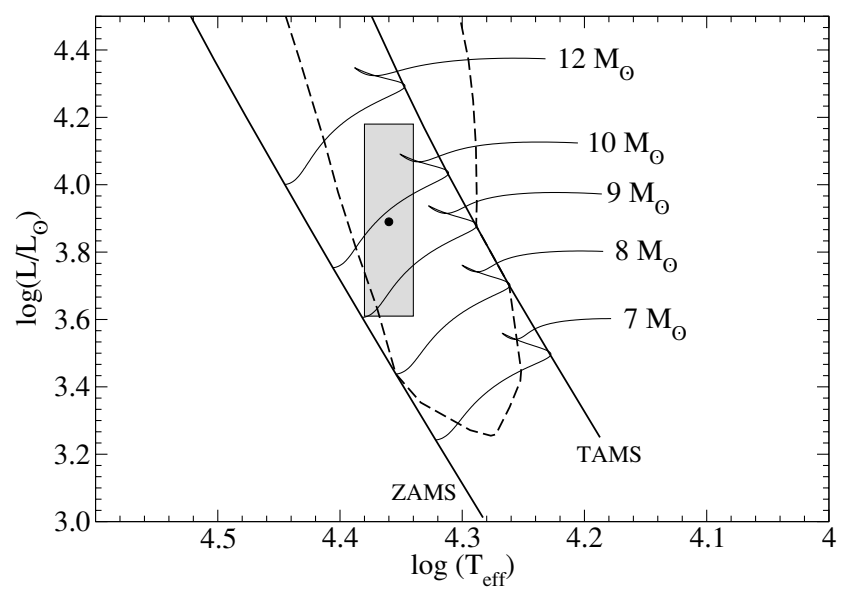

Figure 4. The estimated position and uncertainty box of $v$ Eri in the HR diagram, assuming that $v$ Eri has a solar chemical composition. Synthetic evolutionary tracks are drawn in thin full lines, the zero-age and terminal-age main sequences in thick full lines, and the theoretical $\beta$ Cephei instability strip for $\ell$ up to 2 in a thick dashed line. Except for the evolutionary tracks, which we computed ourselves, the sequences and the strip were taken from Pamyatnykh (1999). They were computed with $X=0.7, Z=0.02$, a mixing length parameter $\alpha=1.0$, and without taking into account the effects of convective core overshooting and rotation. We refer to Pamyatnykh for more details on this instability strip.

$A_{V}$ is very difficult to estimate, we are somewhat fortunate that the Galactic latitude $b=-31^{\circ}$ of $v$ Eri implies that the star does not lie in the patchy Galactic plane, so that we can make a rough estimate of $A_{V}$ with the work of Sandage (1972). This leads to $A_{V} \approx 0.2$, and thus to $V_{0} \approx 3.76$. The Hipparcos parallax $\pi=5.56$ \pm 0.88 mas implies a distance $d=180 \pm 28 \mathrm{pc}$. The absolute visual magnitude is therefore $M_{V_{0}}=-2.52 \pm 0.34$, where we assumed that most of the uncertainty on $M_{V_{0}}$ comes from the uncertainty on the distance rather than from the uncertainty on the dereddened $V_{0}$ magnitude. From the work of Flower (1996) we derive a bolometric correction of $\mathrm{BC}=-2.12 \pm 0.16$, where the uncertainty comes from the uncertainty on $T_{\text {eff }}$. This leads to $M_{\text {bol }}=-4.64 \pm 0.38$ and thus to $\log \left(L / \mathrm{L}_{\odot}\right)=3.76 \pm 0.15$. Another way to estimate the luminosity is to use the strength of the $\mathrm{H} \beta$ Balmer line as measured by the Strömgren $\beta$ index. We applied the $\mathrm{H} \beta$ luminosity calibration of Balona \& Shobbrook (1984), and obtained $M_{V_{0}}=-3.16 \pm 0.40$ and therefore $M_{\text {bol }}=-5.28 \pm 0.43$ and $\log \left(L / \mathrm{L}_{\odot}\right)=4.01 \pm 0.17$, different from but not incompatible with our value obtained with the Hipparcos parallax.

Having two independent estimates for the luminosity, we adopt conservatively the mean value and the union of the error boxes: $\log \left(L / \mathrm{L}_{\odot}\right)=3.89 \pm 0.29$. Deriving $\log \left(L / \mathrm{L}_{\odot}\right)$ from the radius $R=5.1 \pm 0.8 \mathrm{R} \odot$ and the $T_{\text {eff }}=24000 \mathrm{~K}$ of our best FASTWIND model mentioned above, leads to exactly the same value. This is not an independent estimate, however, since the radius for this model was computed with the method of Kudritzki (1980) using the Hipparcos-based $M_{V_{0}}$ derived above. It is nevertheless reassuring that the values are at least consistent. The position of $v$ Eri in the HR diagram with the previously derived $T_{\text {eff }}$ and $\log \left(L / \mathrm{L}_{\odot}\right)$ estimates, is shown in Fig. 4.

\section{MODE IDENTIFICATION}

We used the well-known method of photometric amplitude ratio fitting to determine the degree $\ell$ of each mode (see e.g. Cugier,
Dziembowski \& Pamyatnykh 1994; Balona \& Evers 1999). The observational amplitude ratios (normalized with respect to the $u$ filter) were computed from Table 1 for the nine independent frequencies. To compute the theoretical amplitude ratios, we used the Liège stellar evolution code CLES and the non-adiabatic pulsation code MAD (Dupret 2001) to compute the required non-adiabatic parameters, together with Kurucz (1994) atmosphere models. First we computed a series of models in the error box determined in Section 3. From each of these models we then extracted all modes with $0 \leqslant \ell$ $\leqslant 4$ in the frequency range $[5.5,8] \mathrm{d}^{-1}$, except for the low-frequency mode $v_{10}$ for which we used the frequency range $[0.3,0.55] \mathrm{d}^{-1}$. For each of these modes, we computed the photometric amplitude ratios, and finally we took the average of these ratios and compared them with the observational ratios. To have an idea about the uncertainty of the theoretical amplitude ratios, we also computed the rms scatter, which allows us to define a minimal uncertainty region. The comparison between the theoretical and observational photometric amplitude ratios is shown in Fig. 5. We systematically omitted the theoretical $\ell=3$ amplitude ratios as their peculiar wavelength dependence is easily recognized but not seen in the data. In addition, for $\ell=3$ modes one expects very low light-curve amplitudes due to a considerable surface cancellation effect. From Fig. 5 we see that

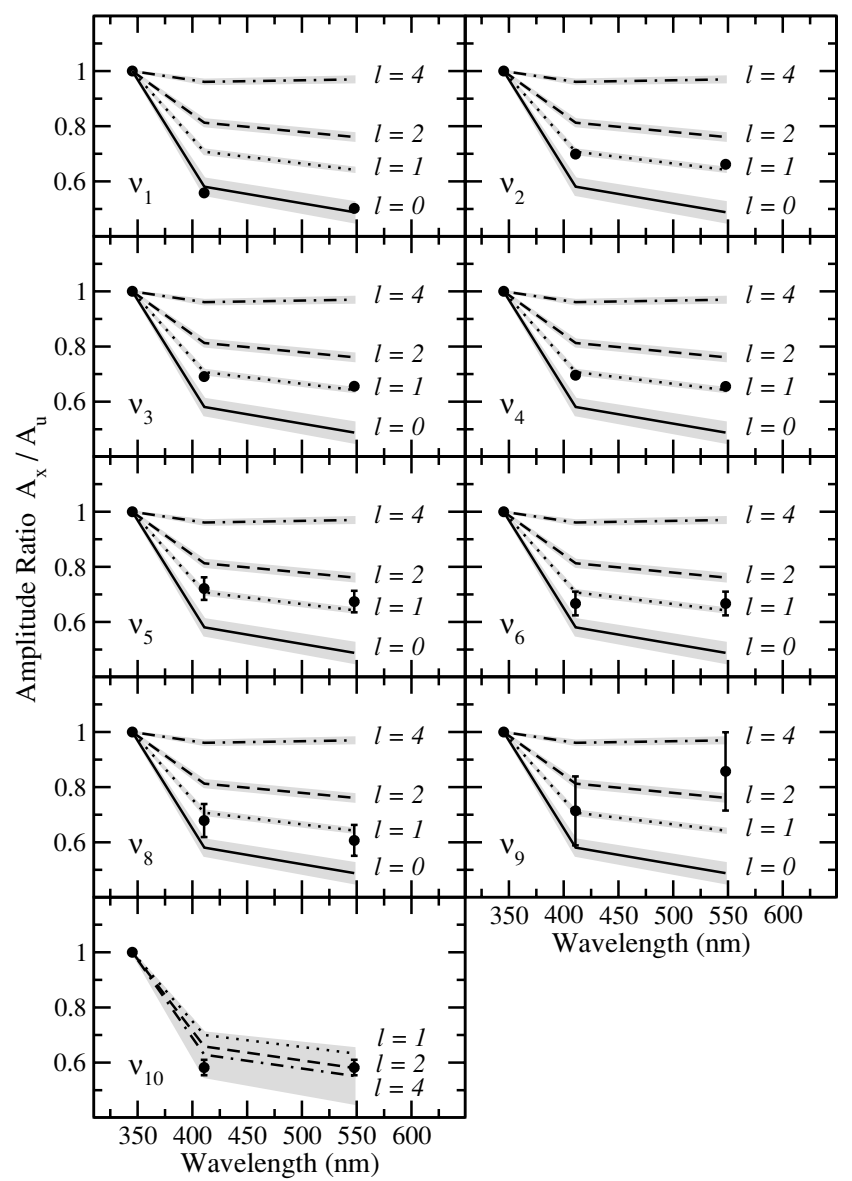

Figure 5. Observed (full circles with error bars) and theoretical $u v y$ amplitude ratios (lines) for $v$ Eri. All amplitudes are normalized with respect to the $u$ filter. Full lines are radial modes, dotted lines are $\ell=1$ modes, dashed lines are $\ell=2$ modes and dashed-dotted lines are $\ell=4$ modes. The grey regions are the minimal uncertainty regions for the theoretical amplitude ratios, computed with the rms scatter of amplitude ratios of the different models in the error box in the HR diagram. The frequency identifications are the same as in Table 1. 


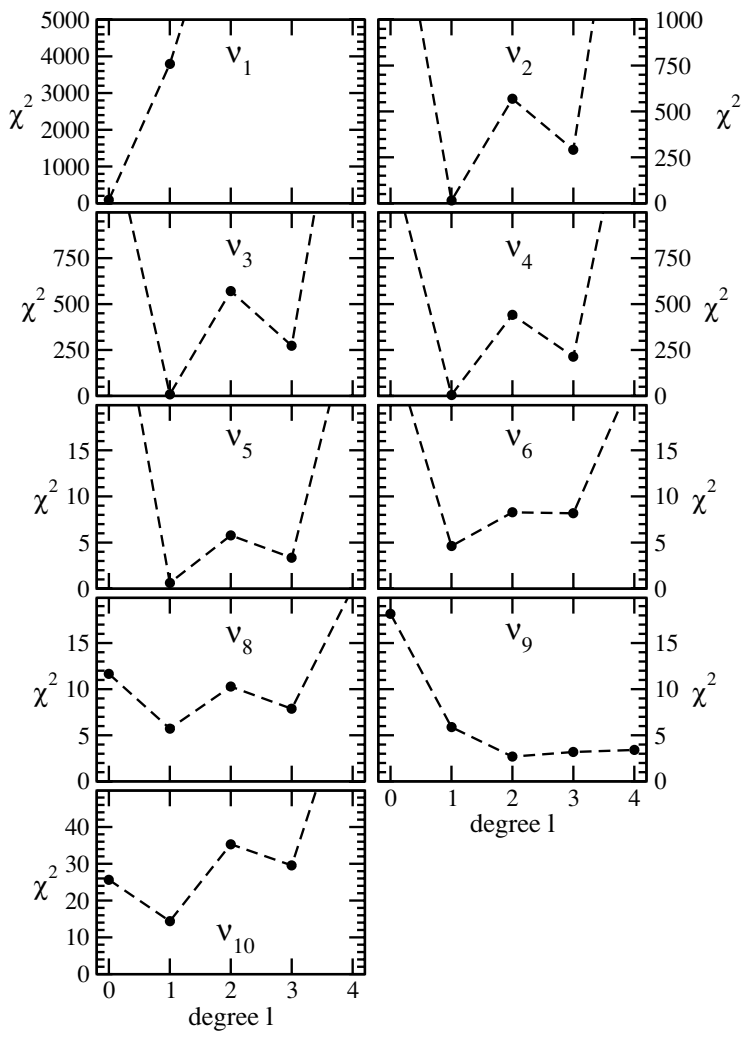

Figure 6. Plots of $\chi^{2}$ as a function of the spherical degree $\ell$ for the model that best fits the observed photometric amplitude ratios and phase differences. This model has parameters $M=9 \mathrm{M}_{\odot}, Z=0.016$ and $T_{\text {eff }}=22500 \mathrm{~K}$. For each mode observed in the photometric time series of Handler et al. (2004), the $\chi^{2}$ value is shown as a function of the degree $\ell$. The frequency identifications are the same as in Table 1 .

$v_{1}$ is clearly a radial mode and that $v_{2}, v_{3}$ and $v_{4}$ are components of an $\ell=1$ triplet. Also $v_{5}$ is clearly an $\ell=1$ mode. For $v_{6}$ and $v_{8}$ there might be some doubt between $\ell=0$ and $\ell=1$, but from theoretical frequency spectra we know that no radial mode can be as close to the radial mode $v_{1}$ as $v_{6}$ and $v_{8}$ are. This implies that $v_{6}$ and $\nu_{8}$ are also $\ell=1$ modes. No clear photometric mode identification is possible for $v_{9}$, as its amplitude is too small. In the panel of the low-frequency mode $v_{10}$, it can be seen that the rms scatter of the theoretical amplitude ratios is rather large. Moreover, this rms scatter is larger for higher degree $\ell$. The uncertainty regions of $\ell=$ 1 and $\ell=2$ are still disjunct, but the uncertainty region of $\ell=4$ overlaps both of them. The comparison with the observational amplitude ratios points towards an $\ell=2$ or an $\ell=4$ mode, although an $\ell=1$ mode cannot be firmly excluded.

The photometric mode identification method outlined above features the characterization of an 'average' radial mode, an 'average' dipole mode, etc. This was done by computing the mean and the rms of theoretical amplitude ratios of all relevant modes of all stellar models in the error box in the HR diagram. We will now adopt a complementary approach where we search for the stellar model that fits best the photometric observations (in the $\chi^{2}$ sense) but without trying to fit the observed frequency values themselves. Besides the photometric amplitude ratios $A_{v} / A_{u}$ and $A_{y} / A_{u}$, we also fitted the photometric phase differences $\varphi_{v}-\varphi_{u}$ and $\varphi_{y}-\varphi_{u}$, and computed the $\chi^{2}$ in the usual way. Given the errors on the photometric amplitudes and phases, the errors on the amplitude ratios and phase differences were computed assuming that measurements in differ- ent passbands are independent. This time the stellar models in the error box in the HR diagram were computed with the Warsaw-New Jersey stellar evolution code, and the non-adiabatic eigenfunctions with the pulsation code of Dziembowski (1977). We also allowed for variations in metallicity $(\in[0.015,0.023])$ but we still neglected the effects of overshooting. The diagrams with $\chi^{2}$ as a function of the degree $\ell$ for the best model are shown in Fig. 6. This model has a mass $M=9 \mathrm{M}_{\odot}$, a metallicity $Z=0.016$ and an effective temperature $T_{\text {eff }}=22500 \mathrm{~K}$. It is reassuring to find essentially the same results as before. The main mode $v_{1}$ is clearly a radial mode and modes $v_{2}$ up to $v_{8}$ and $v_{10}$ are best explained with dipole modes. The frequency $v_{9}$ seems, however, to be best fitted with a degree $\ell$ higher than one.

One of the important differences between the two photometric mode identifications we presented is the selection of the modes. Fig. 5 is based on both stable and unstable modes, while Fig. 6 is based on unstable modes only (except for $v_{10}$, for which we could only find stable modes). Stable modes are usually disregarded for a photometric mode identification, but for $v$ Eri we make an exception. As shown by Pamyatnykh et al. (2004) and by Ausseloos et al. (in preparation), the observed frequency values strangely cannot be explained with standard stellar models if one only considers unstable modes. We therefore included also stable modes for Fig. 5, but disregarded them for Fig. 6 to see to what extent the results would be altered. The important conclusion is that both approaches lead to the same mode identification.

For the modes $v_{1}, \ldots, v_{4}$ and the modes $v_{6}$ and $v_{8}$, we can be fairly confident about their degree $\ell$ as well as their azimuthal order $m$. Of the remaining frequencies for which we still need information, only $v_{7}$ is also present in the spectroscopic data. To gain extra information about this frequency, and about the frequencies 5.51 and $5.89 \mathrm{~d}^{-1}$, we attempted a spectroscopic mode identification by modelling the moments, the amplitude and phase distributions, and the line profiles. For all three techniques we encountered the problem of the enormous amount of computing power required to model reliably and usefully such a large spectroscopic time series containing so many frequencies. Modelling subsets of this time series did not allow the interesting frequencies to be resolved. One of the difficulties is that it is not possible to identify and 'remove' the modes one by one, as the effects of the different modes on the line profiles are coupled, much in contrast with the case of the photometric time series. We actually tried the one-by-one procedure with line profile fitting by binning the line profiles with the frequency with the highest amplitude, then identifying the mode by fitting a one-mode model, and consequently removing the mode by fitting a sinusoid to the line intensity variations at each wavelength and subtracting it from the data. We checked this procedure for the four main modes, for which we already know the wavenumbers, but the results were rather discouraging. To fit the amplitude and phase distributions, we worked initially with a model with four modes. We used both a simple but fast line profile model with a Gaussian intrinsic profile (Schrijvers \& Telting 1999), as well as PULSTAR (De Ridder et al. 2002), which is slower to compute but uses more advanced Kurucz intensity spectra. We did not succeed, however, in finding a reliable fit to the observed amplitude and phase distributions. For $v_{2}, v_{3}$ and $v_{4}$ this is mainly due to the fact that all three modes have a phase distribution with a negative slope (cf. Fig. 2), which would indicate that they are all prograde, which should not be the case for a dipole triplet. From a spectroscopic point of view, the frequencies around $v_{4}$ are more likely explained by three peaks of an $\ell=2$ quintuplet with azimuthal orders $m=0, m=-1$ and $m=-2$. This suspicion was actually already raised by Smith 
(1983), also from a spectroscopic investigation. In this sense there is a clear but unexplained discrepancy between the spectroscopic and the photometric mode identification for the frequencies $v_{2}, v_{3}$ and $v_{4}$.

\section{SUMMARY AND CONCLUSIONS}

We can now summarize the observational results of the 2003 multisite and multitechnique campaign of the $\beta$ Cephei star $v$ Eridani.

According to the photometric mode identification, we find in the radial velocity time series a high-amplitude radial mode $v_{1}$, a dipole triplet $v_{2}, v_{3}, v_{4}$, two close dipole modes $v_{6}$ and $v_{8}$ likely to be part of the same triplet, and yet another dipole mode $v_{5}$ of which we are, however, uncertain whether it is a zonal or a sectoral mode. The degree $\ell$ of the frequency $v_{7}$ could not be determined, and it is therefore uncertain whether it belongs to the same dipole triplet as $v_{6}$ and $v_{8}$. In addition to the frequencies just mentioned, two other significant frequency peaks $\left(v_{9}\right.$ and $\left.v_{10}\right)$ are found in all three observed Strömgren passbands, although we were unable to determine uniquely their degrees $\ell$. Concerning the low-frequency peak $v_{10}$, we explicitly checked again if we could find it in the improved radial velocity time series, but with no positive result. This may cast some doubt upon whether this frequency is indeed a genuine eigenfrequency. The reasons why we included it nevertheless in Table 1 were given in Paper Ia and can be summarized as follows. First, this frequency appears in all three photometric filters. Secondly, its amplitude is far above the noise level. In fact, we find at least three eigenfrequencies with lower amplitudes in the photometry. Thirdly, in Paper Ia it is explicitly checked that the variability at this frequency is not due to the comparison stars $\mu$ Eri or $\xi$ Eri. Fourthly, in the same paper it was also checked that this frequency is not a (reasonable) combination frequency. Finally, fifthly, the frequency is also detectable in the data sets of the individual observatories.

For the rotationally split multiplet around $5.63 \mathrm{~d}^{-1}$ we obtained the following frequency differences: $v_{4}-v_{3}=0.01718(9) \mathrm{d}^{-1}$, $v_{2}-v_{4}=0.01669(7) \mathrm{d}^{-1}$. For the frequencies around $6.24 \mathrm{~d}^{-1}$ we obtained the differences: $v_{8}-v_{6}=0.0171(5) \mathrm{d}^{-1}$ and $v_{6}-v_{7}=$ $0.021(3) \mathrm{d}^{-1}$. The uncertainty on the last-mentioned decimal place is given in parentheses, and was estimated from the scatter between the different values obtained from the different spectral lines and the different Strömgren passbands.

The list of combination frequencies found in the photometric time series is not the same list of combination frequencies found in the improved radial velocity time series, which is in turn not exactly the same as the list of combination frequencies found in the radial velocity time series of Paper $\mathrm{Ib}$, although there is a great deal of overlap in all three lists. The common property of all combination frequencies is, however, that they never include a difference of two independent frequencies.

In the line profiles, a few other candidate frequencies were found in the $\beta$ Cephei frequency range. They are not certain enough to be included in a seismic modelling of $v$ Eri but might be used to gain extra confidence in a model if this model turns out to be compatible with these candidate frequencies.

\section{ACKNOWLEDGMENTS}

$\mathrm{KL}$ and $\mathrm{CA}$ are much indebted to J. Puls for introducing them to the use of the latest version of FASTWIND and for hospitality during their visits to Munich. We thank R. Scuflaire for the use of the stellar evolution code CLES, and M.-A. Dupret for the use of the nonadiabatic pulsation code MAD. We thank W. Dziembowski and A. Pamyatnykh for the use of the Warsaw-New Jersey stellar evolution and pulsation codes. We thank M. Ausseloos for a careful reading of the manuscript. JDR and MB are Postdoctoral Fellows of the Fund for Scientific Research, Flanders. GH's work is supported by the Austrian Fonds zur Förderung der wissenschaftlichen Forschung under grant R12-N02. This study was made possible thanks to the support of the K. U. Leuven Research Fund under grant number GOA/2003/04.

\section{REFERENCES}

Aerts C., 1996, A\&A, 314, 115

Aerts C., de Pauw M., Waelkens C., 1992, A\&A, 266, 294

Aerts C., De Cat P., Cuypers J., Becker S. R., Mathias P., De Mey K., Gillet D., Waelkens C., 1998, A\&A, 329, 137

Aerts C. et al., 2004, MNRAS, 347, 463 (Paper Ib)

Balona L. A., Evers E. A., 1999, MNRAS, 302, 349

Balona L. A., Shobbrook R. R., 1984, MNRAS, 211, 375

Cugier H., Dziembowski W. A., Pamyatnykh A. A., 1994, A\&A, 291, 143

Cuypers J., Aerts C., Buzasi D., Catanzarite J., Conrow T., Laher R., 2002, A\&A, 392, 599

De Ridder J., Dupret M.-A., Neuforge C., Aerts C., 2002, A\&A, 385, 572

Dupret M.-A., 2001, A\&A, 366, 166

Dziembowski W., 1977, Acta Astron., 27, 203

Fitzpatrick E. L., 1999, PASP, 111, 63

Flower P. J., 1996, ApJ, 469, 355

Glushneva I. N., Kharitonov A. V., Kniazeva L. N., Shenavrin V. I., 1992, A\&AS, 92, 1

Handler G., Shobbrook R. R., Jerzykiewicz M. et al., 2004, MNRAS, 347, 454 (Paper Ia)

Hauck B., Mermilliod M., 1998, A\&AS, 129, 431

Herrero A., Puls J., Najarro F., 2002, A\&A, 396, 949

Jamar C., Macau-Hercot D., Monfils A., Thompson G. I., Houziaux L., Wilson R., 1976, Ultraviolet Bright-Star Spectrophotometric Catalogue. A Compilation of Absolute Spectrophotometric Data Obtained With the Sky Survey Telescope (S2/68) on the European Astronomical Satellite TD-1. ESA SR-27, Paris

Kilian J., 1992, A\&A, 262, 171

Kubat J., Puls J., Pauldrach A. W. A., 1999, A\&A, 341, 587

Kudritzki R.-P., 1980, A\&A, 85, 174

Künzli M., North P., Kurucz R. L., Nicolet B., 1997, A\&AS, 122, 51

Kurucz R. L., 1994, Solar Abundance Atmospheres, CD-ROM No 19. Smithsonian Astrophysical Observatory, Cambridge, MA

Leone F., Lanzafame A. C., 1998, A\&A, 330, 306

Mermilliod J.-C., Mermilliod M., Hauck B., 1997, A\&AS, 124, 349

Napiwotzki R., Schönberner D., Wenske V., 1993, A\&A, 268, 653

Pamyatnykh A. A., 1999, Acta Astron., 49, 119

Pamyatnykh A. A., Handler G., Dziembowski W., 2004, MNRAS, in press

Press W. H., Teukolsky S. A., Vetterling W. T., Flannery B. P., 1992, Numerical Recipes in C: The Art of Scientific Computing, 2nd edn. Cambridge Univ. Press, Cambridge

Roberts D. H., Lehar J., Dreher J. W., 1987, AJ, 93, 968

Sandage A., 1972, ApJ, 178, 1

Santolaya-Rey A. E., Puls J., Herrero A., 1997, A\&A, 323, 488

Schrijvers C., Telting J. H., 1999, A\&A, 342, 453

Schrijvers C., Telting J. H., Aerts C., 2004, A\&A, 416, 1069

Smith M. A., 1983, ApJ, 265, 338

Strömgren B., 1966, ARA\&A, 4, 433

Vuille F., 2000, MNRAS, 313, 179

Zboril M., North P., 1999, A\&A, 345, 244

This paper has been typeset from a $\mathrm{T}_{\mathrm{E}} \mathrm{X} / \mathrm{L}_{\mathrm{A}} \mathrm{E} \mathrm{X}$ file prepared by the author. 